\title{
Bevacizumab Combined with Chemotherapy in Children Affected by Hepatocellular Carcinoma: a Single-center Experience
}

\author{
MARIA DEBORA DE PASQUALE ${ }^{1}$, JEAN de VILLE de GOYET ${ }^{2}$, LIDIA MONTI $^{3}$, \\ CHIARA GRIMALDI ${ }^{2}$, ALESSANDRO CROCOLI $^{2}$ and AURORA CASTELLANO ${ }^{1}$ \\ Departments of ${ }^{1}$ Pediatric Hematology/Oncology and Stem Cell Transplantation, \\ ${ }^{2}$ Pediatric Surgery and Transplantation, and ${ }^{3}$ Imaging, Bambino Gesù Pediatric Hospital, Rome, Italy
}

\begin{abstract}
Background: Hepatocellular carcinoma (HCC) is very rare in children and is traditionally associated with a poor prognosis because many patients are not amenable to conventional resection, even in the absence of metastatic disease. For patients with locally advanced or metastatic tumors, conventional chemotherapy appears to offer limited survival benefits. Patients and Methods: We report a case series of five consecutive pediatric patients with HCC who were treated with bevacizumab along with conventional platinum-based chemotherapy. Results: Overall, all five patients presented an objective response to neoadjuvant chemotherapy. Four patients remain alive and free of disease 54 months after diagnosis (range $=20-85$ months) after treatment with bevacizumab in combination with chemotherapy and surgery that consisted of partial liver resection in two patients and liver transplantation in three. Conclusion: In our experience, bevacizumab combined with chemotherapy was an effective and safe option for the treatment of children affected by HCC.
\end{abstract}

Liver tumors account for approximately $1 \%$ of childhood malignancies. Hepatoblastoma (HB) is the most common type, as it accounts for approximately two-thirds of liver tumors in children. Hepatocellular carcinoma (HCC) is the second most common type of liver tumor $(0.5 \%$ of all pediatric malignancies), albeit more common than $\mathrm{HB}$ in areas with endemic hepatitis B virus infection $(1,2)$.

Correspondence to: Maria Debora De Pasquale, MD, Ph.D., Department of Pediatric Hematology/Oncology and Stem Cell Transplantation, Bambino Gesù Children's Hospital, Piazza Sant'Onofrio, 4 - 00165, Rome, Italy. Tel: +39 0668592574, Fax: +390668592292, e-mail: mdebora.depasquale@ opbg.net

Key Words: Hepatocellular carcinoma, childhood, bevacizumab, liver transplantation.
HCC in children may be categorized into two major groups, each with different prognoses. Group 1 includes HCC in diseased liver (e.g. metabolic disease, tyrosinemia, biliary atresia, post-necrotic cirrhosis) with small affected nodes (sometimes diagnosed incidentally) that are resected with the liver since the treatment of primary disease is transplantation (3). The second group includes HCC in healthy liver, which affects children with no previous remarkable history, and is characterized by large-sized masses at diagnosis. For this reason, together with the poor sensitivity of HCC to chemotherapy, a large proportion of children are not amenable to conventional resection, and cure rates are low (estimated at 25-30\%) (4). In the first clinical trial conducted by the International Childhood Liver Tumor Strategy Group (SIOPEL) in 1990, the 5-year overall survival was $28 \%$ for patients with unresectable tumors, and in the last few decades, no change in the outcome of pediatric HCC has occurred $(5,6)$.

The only curative option for pediatric patients is complete surgical resection of the mass, and the only alternative for those with tumors that are not resectable by conventional means is total hepatectomy and liver transplantation (LTx) $(3-5,7)$.

In the latter case, the usefulness of pre-transplant chemotherapy is still under debate. Ismail et al. showed a decrease in alpha fetoprotein (AFP) and regressive changes in tumors of patients who received chemotherapy before LTx (7).

As a matter of fact, the inhibition of angiogenesis represents a potential therapeutic strategy in highly vasculated tumors such as HCC, and many antiangiogenic agents have been approved for the treatment of HCC in adult patients or have entered clinical trials (8). The use of one such agent, bevacizumab, in combination with chemotherapy for the treatment of pediatric relapsed/refractory solid tumors has been reported only in a limited series of patients, anecdotal cases or phase I studies. However, encouraging results have stemmed from such treatment of pediatric patients with HCC 
Table I. Characteristics of the pediatric population under study. Histology was trabecular in all cases.

\begin{tabular}{|c|c|c|c|c|c|c|c|c|c|c|c|}
\hline Case & Gender & $\begin{array}{c}\text { Age } \\
\text { (months) }\end{array}$ & $\begin{array}{l}\text { Intrahepatic } \\
\text { disease }\end{array}$ & $\begin{array}{l}\text { Extrahepatic } \\
\text { extension }\end{array}$ & $\begin{array}{l}\text { AFP at } \\
\text { diagnosis } \\
(\mathrm{ng} / \mathrm{ml})\end{array}$ & $\begin{array}{c}\text { Neoadjuvant } \\
\text { CHT }\end{array}$ & $\begin{array}{l}\text { AFP post } \\
\text { CHT } \\
(\mathrm{ng} / \mathrm{ml})\end{array}$ & Surgery & $\begin{array}{l}\text { Adjuvant } \\
\text { CHT }\end{array}$ & $\begin{array}{l}\text { Relapse/ } \\
\text { progression }\end{array}$ & Status \\
\hline 1 & M & 38 & Monofocal & Lung & 33500 & PLADO & 2097 & Resection & PLADO & Yes & $\mathrm{RC}$ \\
\hline 2 & M & 86 & Monofocal & No & 143510 & PLADO & 3060 & Resection & PLADO & Yes & DOD \\
\hline 3 & M & 98 & Multifocal & No & 110000 & PLADO & 60 & LTx & Oral cycophosphamide & No & $\mathrm{RC}$ \\
\hline 4 & M & 118 & Multifocal & No & 126000 & $\begin{array}{l}\text { PLADO + } \\
\text { GEMOX }\end{array}$ & 1500 & LTx & Oral cycophosphamide & No & $\mathrm{RC}$ \\
\hline 5 & M & 104 & Multifocal & No & 800000 & PLADO & 7000 & LTx & Oral cycophosphamide & No & $\mathrm{RC}$ \\
\hline
\end{tabular}

AFP: Alpha fetoprotein; CHT: chemotherapy; GEMOX: gemcitabine plus oxaliplatin; LTX: liver transplantation; PLADO: cisplatin plus doxorubicin; RC: complete remission; DOD: dead of disease.

$(9,10)$. In this respect, the rarity of disease in the pediatric population explains the difficulty in the design of large clinical trials that might provide definitive therapeutic indications.

At our Institution, in 2009, we decided to treat patients affected by HCC with bevacizumab in addition to chemotherapy due to the dismal prognosis. Here, we report a case series of five consecutive patients.

\section{Patients and Methods}

A review of patients who were treated for HCC from 2009 to 2014 was performed. Data regarding the histopathological subtype of the primary tumor, disease extension, treatment protocol, final status and follow-up were collected and analyzed. Survival of patients, reduction of primary tumor as well as decrease of serum AFP level were considered as study endpoints. All patients underwent an initial pathological diagnosis and AFP measurement. The assessment of the primary tumor was performed by abdominal ultrasound and a computed tomographic (CT) scan of the thorax and abdomen.

After approval for expanded access/compassionate use by the institutional Ethical Committee for every patient in our series and after written informed consent was obtained from the family, bevacizumab was proposed according to a tailored schedule.

All patients were scheduled to receive preoperative chemotherapy, consisting of four blocks of $80 \mathrm{mg} / \mathrm{m}^{2} /$ day cisplatin as continuous intravenous (i.v.) infusion for $24 \mathrm{~h}$ on day 2 and $30 \mathrm{mg} / \mathrm{m}^{2} /$ day doxorubicin as continuous i.v. infusion for 24 hours on days 1 and 3 (PLADO) every three weeks, plus bevacizumab at a dose of $10 \mathrm{mg} / \mathrm{kg}$ every other week.

The respective planning of the surgery and chemotherapy was performed so that the final dose of bevacizumab was administered at least 30 days before the expected date of surgery. For patients listed for transplantation, our protocol activates the waiting list as soon as they recover from the last chemotherapy course, for a period of 3 weeks; if they do not undergo transplantation, they receive another course of chemotherapy while they remain inactive on the waiting list. This protocol can be repeated two to three times.

In patients who benefited from conventional resection, PLADO was continued after surgery in combination with bevacizumab. In patients who underwent transplantation, a metronomic treatment was proposed. This treatment consisted of oral cyclophosphamide $(50 \mathrm{mg}$ on alternate days) and bevacizumab (10 mg/ $\mathrm{kg}$ every other week), together with low-dose tacrolimus monotherapy, which served as an immunosuppressive therapy. Treatment with bevacizumab was restarted no earlier than 6 weeks after surgery.

Tumor response was evaluated before every course of PLADO by measurement of AFP and before the third course by CT scan.

No evident disease (NED) was established when there was both a normal serum level of AFP and a lack of macroscopic primary or metastatic tumor at imaging.

\section{Results}

From 2009 to 2014, five male patients with HCC were diagnosed and treated at Bambino Gesù Pediatric Hospital (Table I) The median age at diagnosis was 98 months (range $=38-118$ months). All patients were affected by the trabecular histopathological subtype of HCC. All patients presented with abdominal swelling as the initial symptom, and two patients also complained of abdominal pain. The AFP level was elevated in all patients at diagnosis (median value $=26,000 \mathrm{ng} / \mathrm{ml}$, range $=33,500-800,000 \mathrm{ng} / \mathrm{ml}$ ).

One patient (case 1) presented with a surgically resectable tumor at diagnosis, but had multiple pulmonary metastases (four in the right lung and six in the left lobe - all with rounded well-defined contours and a predominant subpleural location) (Figure 1A).

In the other four patients, the disease was limited to the liver. In one case, the left lateral section (left lobe according to Couinaud's classification) of the liver was spared and he was considered for conventional resection. In the other three patients (cases 3-5), the tumors were multifocal and were not resectable (Figure 2A); a detailed assessment revealed no vascular invasion or metastatic disease and they were thus considered for LTx.

All patients received preoperative chemotherapy as proposed. At the end of the neoadjuvant treatment period, the tumor response and resectability were re-evaluated by CT scan. At that point, the initial thoughts about resectability were 

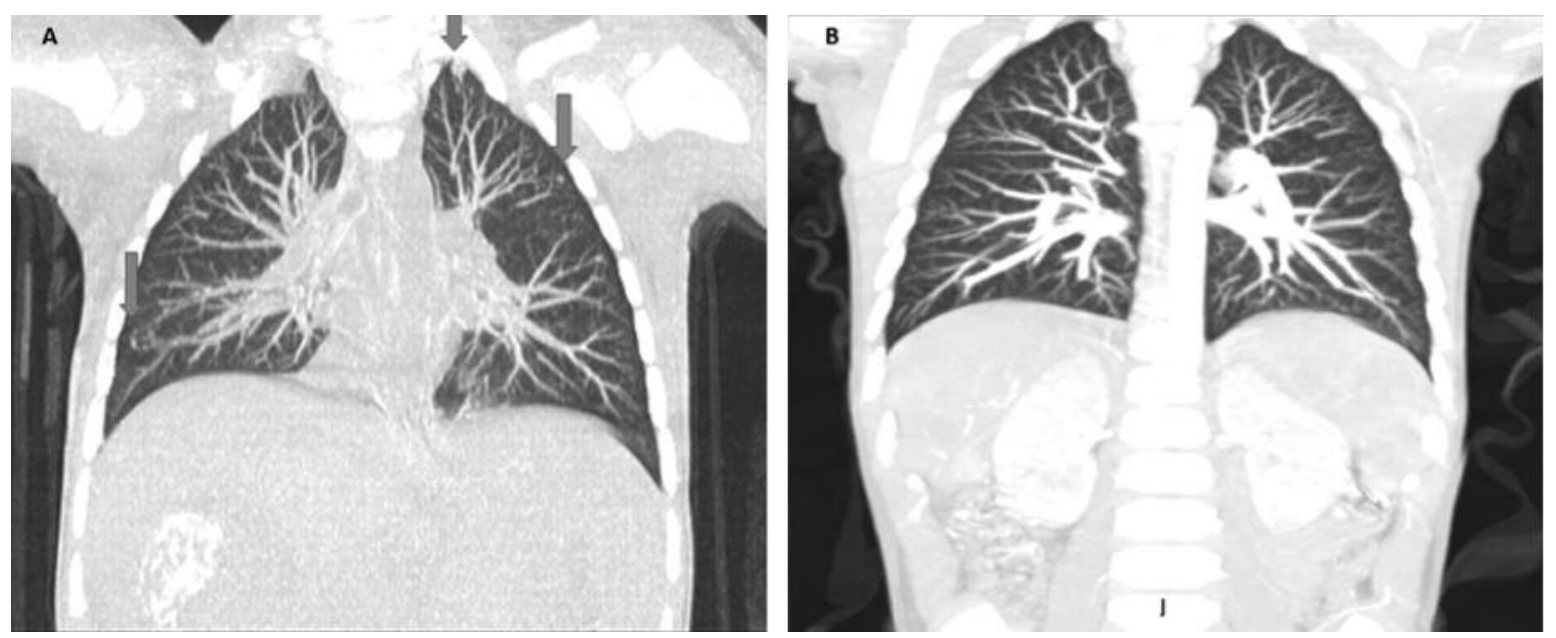

Figure 1. A: Coronal reformatted computed tomographic chest image before (A) and after (B) chemotherapy. A: Pulmonary window shows multiple bilateral peripheral nodules (arrows) consistent with pulmonary metastatic disease. B: Pulmonary window shows no evidence of metastatic disease after chemotherapy.
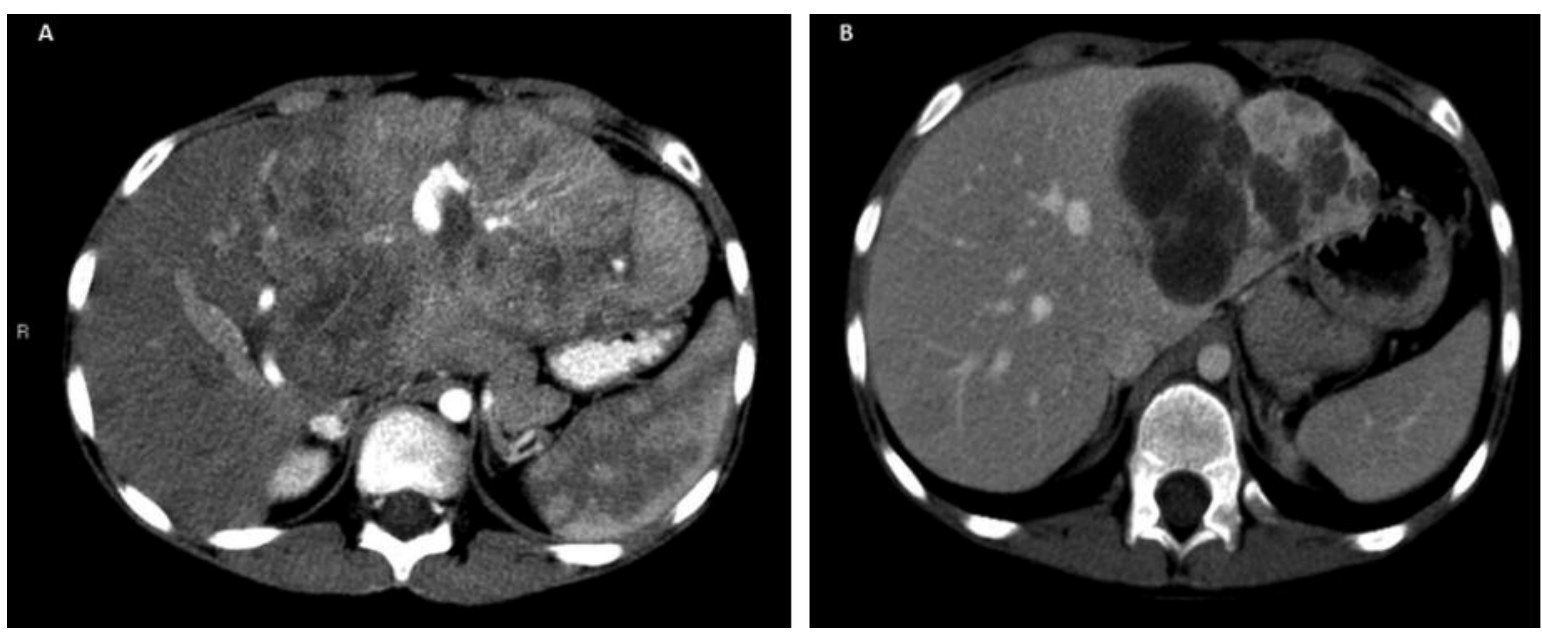

Figure 2. Contrast-enhanced axial computed tomographic of the liver shows a poorly delineated, inhomogeneous enhanced mass with a central star-like scar in the left lobe of the liver (A) before therapy and shows the large space-occupying lesion to have been reduced in size after chemotherapy $(B)$.

confirmed in each case. A reduction in the liver mass was observed in all cases (Figure 2B) and was associated with a regular decrease in the AFP value. Post-chemotherapy presurgical AFP values ranged from 60 to $7000 \mathrm{ng} / \mathrm{ml}$ (median=2097 ng/ml) $($ Table I).

Two patients (cases 1 and 2) underwent partial liver resection (right extended hepatectomy). Patient 1 had a complete remission of pulmonary metastasis after four courses of PLADO plus bevacizumab (Figure 1B) and received two more courses after surgery. Two months after the last course of chemotherapy, and consequent to an increase in AFP, a CT scan revealed a recurrence as a single right pulmonary metastasis. This patient underwent right inferior lobectomy and then began maintenance treatment consisting of oral cyclophosphamide and bevacizumab for 6 months. The AFP level returned to normal 2 months after surgery. This patient is now in the NED category at the end of treatment, at 24 months after diagnosis and 12 months after relapse.

Patient 2 showed an initial decrease in AFP shortly after surgery. The AFP level began to increase again 6 weeks after liver resection. A CT scan showed a recurrence at the level of the lower mediastinum and the right lung ligament, which was resected (regional resection extended to the right inferior segment of the right lung) 9 months after liver surgery. Two 
courses of adjuvant PLADO plus bevacizumab were administered without a reduction in the AFP level. The patient died of disease progression 20 months after diagnosis.

Three patients (cases 3-5) underwent total hepatectomy and subsequent LTx, as planned at diagnosis. One out of these three patients (case 4) received another course of chemotherapy while waiting for LTx because the wait was longer than 3 weeks; this patient received $1,000 \mathrm{mg} / \mathrm{m}^{2}$ gemcitabine with $100 \mathrm{mg} / \mathrm{m}^{2}$ oxaliplatin and underwent a successful transplantation after this extra course. All transplantations involved livers from deceased donors, as follows: one reduced left graft, one left lateral segment and one whole liver graft.

After LTx, maintenance metronomic treatment and immunosuppression were initiated per an established protocol. This was used for an entire year for patient 3. Bevacizumab administration was stopped after 9 months for patient 4 because of grade III hypertension. Patient 5 received this treatment for 3 months but then presented acute graft rejection: this patient demonstrated a poor response to treatment, and the acute rejection evolved into chronic rejection with vanishing bile duct syndrome. The patient was thus considered for re-transplantation, and metronomic treatment was stopped. Currently, the patient is still on the waiting list and is under immunosuppressive treatment with NED at 20 months after diagnosis.

Overall, four patients are currently alive and free of disease at 54 months after diagnosis (range $=20-85$ months).

\section{Discussion}

HCC is very rare in children and is traditionally associated with a poor prognosis $(5,6)$, which is partly secondary to the fact that many patients are not amenable to conventional resection even in the absence of metastatic disease. Traditionally, pediatric patients with HCC have been included in trials that are designed for the treatment of $\mathrm{HB}$ (although $\mathrm{HCC}$ is much less chemosensitive than $\mathrm{HB}$ ) and are not specifically designed for HCC. For locally advanced or metastatic tumors, conventional chemotherapy appears to offer limited survival benefits, and in a recent article presenting the experience of two prospective studies from the SIOPEL group (SIOPEL S2 and SIOPEL S3), intensification of platinum agents does not appear to result in improved survival (6).

In adults, the activity of bevacizumab alone or in combination with cytotoxic agents has been demonstrated in the management of various malignancies (11-15), and several clinical trials have investigated bevacizumab as a treatment for advanced HCC. However, to date, no phase III trials have been conducted (8). Bevacizumab (Avastin ${ }^{\circledR}$; Genentech, Inc.) is a humanized monoclonal neutralizing antibody that binds vascular endothelial growth factor-A (VEGFA)(16). One of the mechanisms that underlies HCC growth is increased tumor angiogenesis caused by heightened expression of VEGF (8, 17). VEGF is overexpressed in HCC, and the level of expression positively correlates with tumor grade (18). Data on the safety and efficacy of bevacizumab in children are limited, but our previous experience has shown that it can be used with reasonably good safety at a dosage of $10 \mathrm{mg} / \mathrm{m}^{2}$ twice a month. In our experience with recurrent and resistant malignancies, we observed satisfactory effects but a relatively high incidence of grade III-IV toxicity, which necessitates careful monitoring during bevacizumab treatment (19).

For the above-mentioned reasons and considering the results of trials in adults with $\mathrm{HCC}$, we decided to treat children diagnosed with $\mathrm{HCC}$ at our center with bevacizumab in addition to chemotherapy. Our goal was not only to be more efficient in the control of the disease and in the reduction of tumor size and expansion, but also to make resection possible as often as possible.

Overall, all five patients presented an objective response to neoadjuvant chemotherapy. Pulmonary metastasis disappeared in patient 1 after four courses of PLADO supplemented with bevacizumab. All patients presented a significant decrease in the level of AFP during neoadjuvant treatment, and all five were subsequently amenable to a radical resection, as all cases regressed to local disease. Overall, the management has been successful, as surgery was proposed for all patients. Four out of the five patients are currently in complete remission with a normalization of AFP level and NED. More interestingly, one patient experienced relapsed 3 months after therapy was completed (single pulmonary metastasis) and was amenable to remission after a second surgery. The overall and relapsefree survival rates were $80 \%$ and $60 \%$, respectively.

Radical tumor resection is the cornerstone of cure for HCC. Since a total hepatectomy is the only way to radically resect large or multifocal HCC in many patients, even when the disease is limited to the liver, LTx is a potentially curative treatment option and an alternative that should be considered. Not only can LTx provide a chance for a cure, but transplantation has come of age and is currently associated with relatively low morbidity and mortality rates due to advances in transplant surgery $(7,20-22)$. In our series, two extended hepatectomies and three transplants were performed, which resulted in excellent outcomes, and all transplanted patients are currently alive. This confirms previous reports that describe the good outcome of LTx in this setting, even when the tumor is relatively large (7). No surgical complications related to antiangiogenic treatment were recorded, not even in those patients who underwent LTx.

In conclusion, in our experience, bevacizumab combined with chemotherapy was an effective and safe option to treat children affected by HCC. Additional evidence continues to show that new chemotherapy regimens will bring new hope to controling HCC. Adult trials that combine neoadjuvant chemotherapy and bevacizumab have shown encouraging results (23), while in pediatric patients, clinicians know that it is a relatively safe drug which can be given in addition to 
chemotherapy $(9,10)$. For this reason, even if the fundamental role of bevacizumab for solid pediatric tumors has not yet been clearly demonstrated, in order to evaluate whether it has an additional effect to that of PLADO, a prospective randomized study, such as an international trial, would be necessary, and further studies that include a larger number of patients are desirable to fully assess the clinical benefits derived from this combination in a pediatric setting.

\section{Conflicts of Interest}

The Authors have no conflicts of interest to disclose.

\section{Financial Support}

The Authors have no financial support to declare.

\section{References}

1 Emre S and McKenna GJ: Liver tumors in children. Pediatr Transplant 8(6): 632-638, 2004.

2 Chen JC, Chang ML, Lin JN, Lai HS, Chen CC, Chen WJ and Hung WT: Comparison of childhood hepatic malignancies in a hepatitis b hyper-endemic area. World J Gastroenterol 11(34): 5289-5294, 2005.

3 Emre S, Umman V and Rodriguez-Davalos M: Current concepts in pediatric liver tumors. Pediatr Transplant 16(6): 549-563, 2012.

4 Allan BJ, Wang B, Davis JS, Parikh PP, Perez EA, Neville HL and Sola JE: A review of 218 pediatric cases of hepatocellular carcinoma. J Pediatr Surg 49(1): 166-171; discussion 171, 2014.

5 Czauderna P, Mackinlay G, Perilongo G, Brown J, Shafford E, Aronson D, Pritchard J, Chapchap P, Keeling J, Plaschkes J, Otte JB and Oncology LTSGotISoP: Hepatocellular carcinoma in children: Results of the first prospective study of the International Society of Pediatric Oncology Group. J Clin Oncol 20(12): 27982804, 2002.

6 Murawski M, Weeda VB, Maibach R, Morland B, Roebuck DJ, Zimmerman A, Casanova M, Perilongo G, Laithier V, Kebudi R, Scopinaro MJ, Shun A, Brichard B, de Camargo B, Childs M, Aronson DC and Czauderna P: Hepatocellular carcinoma in children: Does modified platinum- and doxorubicin-based chemotherapy increase tumor resectability and change outcome? Lessons learned from the SIOPEL 2 and 3 studies. J Clin Oncol 34(10): 1050-1056, 2016.

7 Ismail H, Broniszczak D, Kaliciński P, Markiewicz-Kijewska M, Teisseyre J, Stefanowicz M, Szymczak M, Dembowska-Bagińska B, Kluge P, Perek D, Kościesza A, Dzik E, Lembas A and Teisserye M: Liver transplantation in children with hepatocellular carcinoma. Do Milan criteria apply to pediatric patients? Pediatr Transplant 13(6): 682-692, 2009.

8 Frenette C and Gish R: Targeted systemic therapies for hepatocellular carcinoma: Clinical perspectives, challenges and implications. World J Gastroenterol 18(6): 498-506, 2012

9 Venkatramani R, Malogolowkin M, Davidson TB, May W, Sposto $\mathrm{R}$ and Mascarenhas L: A phase i study of vincristine, irinotecan, temozolomide and bevacizumab (vitB) in pediatric patients with relapsed solid tumors. PLoS One 8(7): e68416, 2013.

10 Wagner L, Turpin B, Nagarajan R, Weiss B, Cripe T and Geller $\mathrm{J}$ : Pilot study of vincristine, oral irinotecan, and temozolomide (VOIT regimen) combined with bevacizumab in pediatric patients with recurrent solid tumors or brain tumors. Pediatr Blood Cancer 60(9): 1447-1451, 2013.

11 Yang JC, Haworth L, Sherry RM, Hwu P, Schwartzentruber DJ, Topalian SL, Steinberg SM, Chen HX and Rosenberg SA: A randomized trial of bevacizumab, an anti-vascular endothelial growth factor antibody, for metastatic renal cancer. N Engl J Med 349(5): 427-434, 2003.

12 Welch S, Spithoff K, Rumble RB, Maroun J and Group GCDS: Bevacizumab combined with chemotherapy for patients with advanced colorectal cancer: A systematic review. Ann Oncol 21(6): 1152-1162, 2010.

13 Friedman HS, Prados MD, Wen PY, Mikkelsen T, Schiff D, Abrey LE, Yung WK, Paleologos N, Nicholas MK, Jensen R, Vredenburgh $J$, Huang $J$, Zheng $M$ and Cloughesy $T$ : Bevacizumab alone and in combination with irinotecan in recurrent glioblastoma. J Clin Oncol 27(28): 4733-4740, 2009.

14 Thomas MB, Morris JS, Chadha R, Iwasaki M, Kaur H, Lin E, Kaseb A, Glover K, Davila M and Abbruzzese J: Phase ii trial of the combination of bevacizumab and erlotinib in patients who have advanced hepatocellular carcinoma. J Clin Oncol 27(6): 843850, 2009.

15 Perez EA, Hillman DW, Dentchev T, Le-Lindqwister NA, Geeraerts LH, Fitch TR, Liu H, Graham DL, Kahanic SP, Gross HM, Patel TA, Palmieri FM and Dueck AC: North central cancer treatment group (NCCTG) n0432: Phase II trial of docetaxel with capecitabine and bevacizumab as first-line chemotherapy for patients with metastatic breast cancer. Ann Oncol 21(2): 269-274, 2010.

16 Presta LG, Chen H, O'Connor SJ, Chisholm V, Meng YG, Krummen L, Winkler M and Ferrara N: Humanization of an antivascular endothelial growth factor monoclonal antibody for the therapy of solid tumors and other disorders. Cancer Res 57(20): 4593-4599, 1997.

17 Sia D and Villanueva A: Signaling pathways in hepatocellular carcinoma. Oncology 81(Suppl 1): 18-23, 2011.

18 Li XM, Tang ZY, Zhou G, Lui YK and Ye SL: Significance of vascular endothelial growth factor mRNA expression in invasion and metastasis of hepatocellular carcinoma. J Exp Clin Cancer Res 17(1): 13-17, 1998.

19 de Pasquale MD, Castellano A, de Sio L, de Laurentis C, Mastronuzzi A, Serra A, Cozza R, Jenkner A and de Ioris MA: Bevacizumab in pediatric patients: How safe is it? Anticancer Res 31(11): 3953-3957, 2011.

20 Moore SW, Hesseling PB, Wessels G and Schneider JW: Hepatocellular carcinoma in children. Pediatr Surg Int 12(4): 266270, 1997.

21 Chen JC, Chen CC, Chen WJ, Lai HS, Hung WT and Lee PH: Hepatocellular carcinoma in children: Clinical review and comparison with adult cases. J Pediatr Surg 33(9): 1350-1354, 1998.

22 Tagge EP, Tagge DU, Reyes J, Tzakis A, Iwatsuki S, Starzl TE and Wiener ES: Resection, including transplantation, for hepatoblastoma and hepatocellular carcinoma: Impact on survival. J Pediatr Surg 27(3): 292-296; discussion 297, 1992.

23 Fang P, Hu JH, Cheng ZG, Liu ZF, Wang JL and Jiao SC: Efficacy and safety of bevacizumab for the treatment of advanced hepatocellular carcinoma: A systematic review of phase II trials. PLoS One 7(12): e49717, 2012.

Received January 2, 2017

Revised February 9, 2017

Accepted February 13, 2017 